\title{
POUR UNE SOCIOLOGIE UTILE : LA SOCIOLOGIE D'INTERVENTION
}

\author{
Denis Bernardeau Moreau \\ Université Paris-Est Marne la vallée, laboratoire ACP \\ 5 bd René Descartes Champs-sur-Marne 77454 Marne-la-Vallée Cedex2 \\ denis.bernardeau@univ-mlv.fr
}

\section{FOR AN USEFUL SOCIOLOGY: THE SOCIOLOGY OF INTERVENTION}

\section{Résumé :}

Poser la question de l'utilité de la sociologie nous conduit à interroger les rôles, postures et missions que se donnent les sociologues pour faire avancer les connaissances sur les situations et les faits sociaux. Parmi ces postures, nous voulons évoquer en particulier celle du scientifique se réclamant de la sociologie d'intervention. La sociologie d'intervention est une pratique de la discipline conduisant le sociologue à s'immerger dans une situation locale, afin d'agir sur elle. Se positionnant comme un tiers, rapprochant, autant que possible, les savoirs savants et les savoirs «profanes », le sociologue d'intervention se place entre les individus pour les aider à mieux gérer leur vie en collectivité. Sa posture répond à un souci d'utilité.

Mots clés : Intervention, maïeutique, immersion, sens commun, utilité.

\section{Abstract :}

Asking the question of the usefulness of sociology leads us to question the roles, missions and postures that will give social scientists to advance their knowledge about situations and social facts. Among these positions, we want to mention in particular the sociologist practitioner claiming the sociology of intervention. The sociology of intervention is a practical discipline leading sociologist to immerse themselves in a local situation to improve it. Acting as a third party, the sociologist intervention takes place between individuals to help them better manage their community life. By bringing as much as possible, academic knowledge and popular knowledge, it contributes to the well-being of individuals in their community life. The sociologist raises the question of his usefulness.

Keywords: Intervention, midwifery, immersion, common sense, utility. 
«A quoi sert la sociologie? Quelles sont ses finalités pratiques? Les sociologues ont-ils une utilité en dehors de leur vocation à proposer un maniement de l'esprit critique?». Telles sont les questions que se posent des sociologues comme Michel Crozier, Gilles Herreros, Bernard Lahire et que nous souhaitons aborder à notre tour comme point de départ de notre réflexion. Poser la question de l'utilité de la sociologie nous conduit à interroger les rôles, postures et missions que se donnent les sociologues pour faire avancer les connaissances sur les situations et les faits sociaux. Notre cheminement, à la fois intellectuel, scientifique et empirique, nous renvoie sans cesse à la question de notre mission et à l'impact de nos recherches dans la vie professionnelle et quotidienne des individus. Notre intention, dans cet article, n'est pas de traiter des clivages idéologiques qui animent régulièrement les débats scientifiques et en particulier la communauté des sociologues. Notre intention est plutôt d'expliciter notre préférence pour une sociologie d'intervention. De ce point de vue, l'utilité de nos recherches se mesure en partie aux effets bénéfiques directs qu'elles peuvent produire sur les acteurs du monde social.

\section{LE SOCIOLOGUE ENTRE DISTANCIATION, INTERVENTION ET ENGAGEMENT}

\subsection{LES TROIS POSTURES DU SOCIOLOGUE}

Les questionnements, portant sur la posture et l'utilité du sociologue, reviennent souvent dans les débats et rendent compte de perceptions parfois peu conciliables. Comme le rappelle Marc Uhalde (2001, p. 462-463 et 2008, p. 97-98), il est possible d'identifier globalement trois figures du sociologue, distinctes mais complémentaires: le sociologue scientifique, le sociologue opérationnel et le sociologue critique ${ }^{1}$. La première définition est donnée par Max Weber et Pierre Bourdieu. Au sens wébérien, le sociologue est un scientifique dont l'œuvre est de faire progresser la science pour la science en dehors de tout intérêt politique et économique. Son action relève de la rationalisation intellectuelle. Savant mais pas politique, il

\footnotetext{
${ }^{1}$ Notons également la classification proposée par Jean Dubost en fonction de la sensibilité du sociologue. Selon que le sociologue est plutôt sensible à la recherche, il tend à adopter une posture d'indépendance en donnant la priorité à la méthode. S'il est sensible aux acteurs, sa position est davantage celle du tiers visant à répondre en priorité aux problèmes internes. Si sa sensibilité va aux décideurs, il se fait opérateur soucieux de respecter le cahier des charges des commanditaires. Enfin, si le sociologue est davantage sensible au système, il se fait critique en donnant priorité à l'action militante (Dubost, 2006, p.146).
} 
doit mettre de côté ses jugements de valeur s'il veut atteindre une compréhension objective des faits. Dans la définition que propose Pierre Bourdieu, le sociologue, attaché à la neutralité axiologique, établit une rupture avec le sens commun. Fort de sa capacité de distanciation que lui procure sa position savante (Uhalde, 2001, p. 462), il est un producteur de connaissances scientifiques sur le monde social. La deuxième figure du sociologue est celle du praticien, privilégiée par des auteurs comme Gilles Herreros, Alain Touraine ou encore Michel Wieviorka. La posture qu'il donne à voir est celle du sociologue opérationnel. «Cette définition du métier de sociologue met en avant la fonction interventionniste de la sociologie et son utilité au sein et pour les organisations » (Piriou, 1999, p. 110). Tel un «clinicien » (le mot vient de Klinos signifiant lit ou chevet du malade), le sociologue tente de répondre à la demande sociale en analysant les organisations de l'intérieur et en aidant les individus à mieux comprendre les ressorts de leurs actions. Il est un praticien ayant acquis une très bonne maîtrise des outils et connaissances sociologiques et psychosociologiques. Le sociologue intervient comme «révélateur» des difficultés relationnelles entre les acteurs et comme «animateur» des moyens d'y remédier (Alter, 1995, p. 143-144). Pour d'autres enfin, le sociologue est un militant engagé dans la défense d'une cause au nom de valeurs qui l'habitent et guident son analyse. Insérant délibérément son activité d'intellectuel dans le combat politique, le sociologue critique assume ses propres jugements de valeur dans son interprétation des phénomènes sociaux. A l'image des tenants de l'analyse institutionnelle (Lourau, Lapassade, Hess), il s'agit de dénoncer les rapports de domination et les inégalités sociales qu'ils génèrent. Remarquons toutefois qu'il convient de relativiser ces notions d'objectivité et d'engagement dans les sciences sociales car l'histoire des sociologues, comme l'explique bien Gilles Herreros, souligne à l'évidence la difficulté de ces derniers à être dans une complète distanciation avec leurs objets étudiés. «La production sociologique n'est jamais un simple regard, neutre et distant, porté sur son objet (...). Cette activité, pour savante qu'elle se revendique et se définisse, n'en est pas moins intellectuellement engagée (Herreros, 2009, p. 229). En sociologie comme ailleurs, l'objectivité peut être un but mais il est rarement atteint car, comme le dit Bourdieu, «le sociologue ne peut ignorer que le propre de son point de vue est d'être un point de vue sur un point de vue» (1993, p.1423), une connaissance de connaissances.

\subsection{L'UNIVERSITAIRE ET LE PROFESSIONNEL : DEUX UNIVERS QUI SE RAPPROCHENT}


De ces trois positionnements, revenons sur les deux premiers. Parmi les nombreux qualificatifs dont la littérature sociologique se nourrit pour décrire le métier de sociologue, deux registres sont nettement distingués. Les sociologues académiques exercent dans le champ de la recherche publique (université, CNRS, INED etc.), tandis que les sociologues praticiens travaillent en entreprise. Même si des tentatives de rapprochement ont été proposées notamment par Renaud Sainsaulieu et Monique Legrand (1995), les clivages perdurent. A la différence du sociologue académique qui, lui, «fait référence à la communauté savante »(Piriou, 2006, p. 15) et exerce dans le milieu de la recherche, le sociologue praticien est davantage un "diplômé de haut niveau en sociologie qui utilise les connaissances de sociologie, en dehors de l'université et de la recherche publique ${ }^{2}$ (Ibid., p.14). Si, en différenciant ces deux profils de sociologue ${ }^{3}$, on peut craindre une stigmatisation entre deux manières de pratiquer la sociologie, Odile Piriou soulève un problème de fond sur l'influence du contexte institutionnel dans la définition des conditions de l'exercice professionnel. Jusqu'à peu, l'universitaire tendait à incarner préférentiellement le chercheur attaché à une réflexion conceptuelle, cultivant son indépendance et sa liberté de pensée et analysant «d'en haut» les pratiques quotidiennes des individus en société, tandis qu'à l'opposé, le praticien était l'expert et l'homme de terrain utilisant à des fins professionnelles les outils de mesure de l'activité sociale. Or, depuis une bonne décennie, l'université connaît une évolution importante qui l'oblige à reconsidérer cette dichotomie. Dans une situation économique difficile pour les jeunes salariés, l'université doit repenser ses diplômes et sa capacité à optimiser les chances d'insertion professionnelle de ses étudiants. «Si les préoccupations des milieux universitaires, note Renaud Sainsaulieu, restent centrées sur la transmission d'une compétence scientifique, pour être crédibles aux yeux de leurs étudiants, ils doivent prouver la valeur de leurs enseignements par leur possibilité de mise en ouvre concrète » (1995, p. 24). Dans un rapport d'enquête de 2005 portant sur le «bouleversement de la table des valeurs académiques» des enseignants chercheurs, Sylvia Faure, Charles Soulié et Mathias Millet décrivent longuement le clivage profond qui se crée aujourd'hui

\footnotetext{
${ }^{2}$ Pour Odile Piriou, le sociologue académique est classiquement un «scientifique pur » ayant validé des études supérieures en sociologie mais ne disposant pas de réelle expérience professionnelle en dehors de l'université. Le sociologue praticien est davantage un «converti » globalement moins diplômé mais disposant d'une forte expérience professionnelle mobilisant ses savoirs théoriques et pratiques.

${ }^{3}$ Pour distinguer ces deux sociologies, Hess compare les deux ouvrages de Montesquieu : «L'esprit des lois » qui relève, écrit-il, de la « sociologie abstraite » et «Les lettres persanes » qu'il attribue à une « sociologie de terrain » (Hess, 1981, p. 59-60).
} 
entre les enseignants chercheurs académiques et ceux épousant un profil plus «managérial». Aux «experts scientifiques» s'ajoutent désormais des «experts en organisations universitaires » tout autant directeurs de filières qu' «entrepreneurs » de leurs formations (Dubet, 2003, p. 377). George Félouzis compare même ses nouveaux directeurs à des «petits entrepreneurs indépendants»(2003). Aujourd'hui, 1'universitaire multiplie les passerelles avec le monde extérieur pour mieux adapter ses enseignements et ses formations. Dans un espace de plus en plus concurrentiel ${ }^{4}$, il gère ses formations et côtoie le chargé de mission ou le professionnel partageant son temps entre son activité en entreprise et ses cours à l'université. D'après le rapport précédemment cité, on est ainsi passé, entre 1992 et 2002, de 400 à plus de 3000 postes de PAST (les profils correspondants de ces professeurs associés et invités étant en général celui de cadres de l'industrie des secteurs public et privé).

Sans aucun doute, cette ouverture est inéluctable, mais elle présente aussi un risque réel : celui d'une perte d'indépendance de l'universitaire dès lors que ses maquettes de formation et ses enseignements deviennent quelque peu tributaires de l'évolution à court terme du marché de l'offre et de la demande. L'autonomie du champ scientifique a été fortement défendue par quelques grands sociologues tels Pierre Bourdieu qui y voyait une condition pour préserver la science d'un risque d'instrumentalisation. Plus un champ est autonome et plus il est légitime pour critiquer les phénomènes sociaux qui l'entourent, écrivait-il. Demandant à tous les chercheurs quels qu'ils soient de dépasser leurs propres clivages, Pierre Bourdieu appelait de ses vœux l'affirmation d'une science «pure » régie par un pouvoir scientifique contre une science «serve» gouvernée par un pouvoir institutionnel et politique (Bourdieu, 1997, notamment p. 15 et p. 65). Ce risque s'asservissement est réel et témoigne de la difficulté, pour le sociologue, à s'entendre sur la finalité de son travail. Doit-il préserver le monde du savoir des contingences extérieures et garantir coûte que coûte l'autonomie de sa science ou doit-il, au contraire, s'ouvrir au monde «profane $»^{5}$, au risque de «perdre un peu de son âme » et de son indépendance d'esprit dans une dilution possible du projet scientifique ? C'est

\footnotetext{
${ }^{4}$ Outre une concurrence de plus en plus affichée avec les écoles privées, l'université subit aussi une concurrence très forte de ses filières courtes, plus professionnelles et, aux dires des étudiants, moins incertaines. On pense bien entendu aux IUT, DUT et BTS dont les débouchés, peut-être modestes en termes de responsabilités et de rémunérations, ont l'avantage de la transparence et de la clarté. En aspirant à un meilleur encadrement institutionnel durant leur formation, les étudiants affichent leur «aversion croissante pour le risque » (Veltz, 2007, p.52) dans un monde du travail difficile, voire anxiogène.

${ }^{5}$ Le profane est celui qui n'est pas initié, celui qui n'appartient pas à la sphère du savoir, c'est-à-dire aux institutions dûment consacrées.
} 
la difficile question de la finalité de son métier que le sociologue est amené sans cesse à se poser.

\section{L'UTILITE DE LA SOCIOLOGIE PASSE AUSSI PAR L'INTERVENTION}

\subsection{LE SOCIOLOGUE FACE A LA DEMANDE SOCIALE}

Excluant une posture jugée trop théorique et intellectuelle quand elle n'est vouée qu'à «une esthétique de la réflexion» (Herreros, 2009, p.8), certains sociologues affirment leur préférence pour le singulier plutôt que la globalité, les situations concrètes plutôt que les grandes théories sociales. Dans son livre «A quoi sert la sociologie?», Michel Crozier préconise ainsi le recours aux études de cas «cliniques » pour mieux appréhender la société. «Le chercheur n'est pas supposé prédire l'avenir, ni fournir une appréciation définitive des modes de fonctionnement existants et encore moins d'élaborer tout seul le nouveau modèle d'organisation. Tout ce que l'on devrait attendre de lui est la production de connaissances empiriques sur la structure et les modes de régulation réels du système humain qui soustendent un champ d'action donné »(Crozier, 2000, tome 1, p. 157). La démarche du sociologue, poursuit Gilles Herreros, doit être une pratique «dont les perspectives sont éminemment pragmatiques et dont les effets sont tangibles »(Herreros, 2009, p. 8). Ainsi, loin d'une sociologie parfois très abstraite, certains sociologues optent résolument pour une posture plus opérationnelle et interventionniste. Eloignée des débats épistémologiques et des querelles de méthodes, cette sociologie dont se réclament ces chercheurs, défend l'idée de son opérationnalité. En cela, elle est une sociologie d'intervention, telle que l'ont définie, chacun à leur manière, Jean Dubost, Gilles Herreros, Rémi Hess, Antoine Savoye ou encore Alain Touraine. Aujourd'hui, il est indéniable qu'«une autre forme de compétence pratique opératoire s'impose au métier de sociologue», écrit Sainsaulieu (1995, p. 21). Cette compétence, c'est sur le terrain que le sociologue l'acquiert par une observation précise et une immersion réelle en situation. Intrinsèquement pratique, cette sociologie impose de la rigueur dans le recueil des données, une démarche résolument cognitive qui contrevient à l'idée parfois répandue qu'elle serait «un lieu vide où ne s'exprimeraient que des sociologues de seconde zone »(Vrancken, 2001, p. 311).

Face à des situations où les interactions entre les individus sont chaque fois particulières, où les contextes donnent à voir des trajectoires et des histoires de vie singulières, notre rôle de sociologue s'inscrit donc dans une quête de sens et d'utilité. Le sens est la visée subjective 
qu'un individu donne à son action, en fonction de sa biographie, de son parcours personnel et professionnel, de son expérience, de sa culture. L'utilité se situe, de notre point de vue, dans les réponses que le sociologue est capable d'apporter aux demandes exprimées plus ou moins formellement par les individus évoluant dans les organisations sociales. La demande sociale relève de sources multiples : elle peut émaner d'une commande institutionnelle publique (collectivités territoriales, organismes d'Etat) ou provenir d'entreprises privées pour des activités de conseil, de formation ou d'aide à la décision. Il peut s'agit de répondre à des sollicitations d'acteurs divers connaissant des difficultés dans leurs organisations professionnelles. La demande peut enfin provenir de collègues ou de partenaires universitaires, syndicaux ou encore associatifs (communications, colloques, journées d'études, conférences). Ainsi définie, l'utilité du sociologue d'intervention se mesure essentiellement à la capacité de ce dernier à agir directement sur les situations quotidiennes des individus. Ce sociologue doit savoir adopter une démarche empirique en s'immergeant régulièrement dans le milieu qu'il étudie, pour répondre, comme le rappelle Robert Castel, à la demande sociale entendue comme «le système d'attente de la société à l'égard des problèmes quotidiens qui la sollicitent aujourd'hui » (2004, p. 70). Ces liens entre sociologie et demande sociale sont essentiels. Ils sont même, ajoute Dubar, décisifs pour l'avenir de la discipline. Plus ces liens seront forts et plus la sociologie sera reconnue et efficace (Dubar, 2006, p. 11).

De tous les courants qui se réclament de cette utilité sociale, la sociologie d'intervention en est, de notre point de vue, une forme particulièrement intéressante. La posture du sociologue est celle du tiers plus que du visionnaire, celle du médiateur plus que de l'observateur, aidant les individus engagés sur le terrain à mieux comprendre le monde dans lequel ils vivent.

\subsection{LE SOCIOLOGUE EN IMMERSION A L'ECOUTE DES INDIVIDUS REFLEXIFS}

Parmi les écoles qui se revendiquent clairement de la sociologie de l'intervention, le corpus classique comprend trois principaux courants : l'actionnalisme d'Alain Touraine (dont se réclament aussi François Dubet et Michel Wieviorka) se donne pour but d'analyser les mouvements sociaux en tant qu'expression de l'action des hommes désireux de maîtriser leur historicité. Dans une dimension plus clinique, il y a l'analyse stratégique de Michel Crozier et Erhard Friedberg qui développe une pensée originale construite sur les jeux de pouvoirs entre acteurs rationnels et stratégiques. Il y a également l'analyse institutionnelle de Rémi Hess, 
George Lapassade et René Lourau qui exprime une volonté de rupture avec un certain conformisme scientifique ${ }^{6}$. Rangés dans un courant plus psychosociologique et clinique, on trouve également les travaux d'Eugène Enriquez, Vincent De Gaulejac et Nicole Aubert ${ }^{7}$.

La sociologie d'intervention, explique Guy Minguet, est un exercice professionnel, épistémologique, méthodologique, postural qui conduit le sociologue praticien à se positionner au cœur d'une situation afin de l'améliorer (Minguet, 2001, p. 21). Le sociologue doit s'immerger dans la situation qu'il analyse. Par le mot « intervention », il s'agit de penser le sociologue comme un scientifique capable de faire évoluer, avec les acteurs objets et sujets de l'étude, les situations dans lesquelles ils interagissent pour atténuer collectivement les difficultés qu'ils rencontrent. Felder compare très justement l'immersion à l'acte de se laisser « imprégner par les impressions » (2007, p.242). Il s'agit de privilégier un rapport immédiat, direct et intersubjectif entre le chercheur et les acteurs. En partant le plus souvent d'un problème posé par les demandeurs, l'intervention marque une action tournée vers le changement. Par sociologie d'intervention, il faut donc entendre «une pratique spécifique de la discipline conduisant le sociologue à s'immerger, sur (auto)sollicitation, dans une microsituation, afin d'en proposer une lecture dont les effets (de contenu ou de processus) pourraient participer d'une transformation de ladite situation» (Herreros, 2001, p. 273). La posture du sociologue consiste dès lors à se placer parmi les individus en situation, dans l'intention de leur apporter «une plus-value cognitive mobilisable pour l'action » (Ibid.). En fait, toute sociologie peut relever de la posture d'intervention dès lors qu'il s'agit (et cela arrive à chacun d'entre nous un jour ou l'autre) de confronter les conclusions d'une enquête avec les points de vue des acteurs concernés sur le terrain. Pour autant, cette confrontation peut être de nature différente, directe ou sous-jacente. Comme le souligne François Dubet, dans la nature des relations entre le chercheur et les individus, «tout est affaire de degré » (Dubet, 2001, p. 90). La sociologie d'intervention trouve donc sa raison d'être dans une

\footnotetext{
${ }^{6}$ En réalité, les origines de la sociologie d'intervention sont plus lointaines, à la frontière entre sciences sociales et sciences humaines. D'un point de vue sociologique, on peut, avec Savoye (1979) et Hess (1981), en situer les prémices dans la microsociologie monographique de Le Play, relayée par les sociologues empiristes américains notamment de l'école de Chicago. Ce n'est qu'ensuite que la sociologie européenne prend le relai (voir à ce sujet notre article à paraître en 2014).

${ }^{7}$ Dans sa très large taxinomie des modèles sociologiques d'intervention, Guy Minguet (2001) propose au total une dizaine de modèles. Dans ce catalogue très hétéroclite, figurent en plus des théories déjà citées le courant de l'école des relations humaines, l'approche sociotechnique ou encore le modèle de la contingence structurelle. Notons que ces courants ne se réclament pas tous du courant interventionniste.
} 
relation privilégiée et approfondie entre le chercheur et l'ensemble des individus en vue de faire évoluer une situation. Aux théories scientifiques du sociologue doivent répondre les «théories spontanées » (Dubet, 1994, p. 232) des acteurs qui sont experts de leur terrain. Cela signifie également que la sociologie d'intervention considère l'individu comme l'acteur réflexif essentiel de sa propre évolution (Dubost, 1987, p.178). Reconnaître la réflexivité de l'acteur, c'est reconnaître qu'il possède l'intelligence de son action. C'est affirmer qu'il est compétent pour porter un regard sur ce qu'il fait, pour mesurer les conditions d'émergence et en partie les conséquences de ses actions. Pour Giddens (1987), cette réflexivité revêt même un usage systématique. L'auteur de la théorie de la structuration distingue ainsi deux types de réflexivité. La réflexivité qui s'inscrit dans la «conscience pratique »(Giddens, 1987, p.92) correspond au contrôle minimal que l'individu est capable d'effectuer, dans l'ensemble de sa vie quotidienne et routinière, sur son action en train de se faire ${ }^{8}$. A un niveau supérieur, la réflexivité, chez Giddens, renvoie à la «conscience discursive ». Cette conscience s'exerce quand l'individu parvient à exposer de manière cohérente les raisons de son action, quand il peut «mettre en mots des choses»(Giddens, 1987, p.93). Dans la mesure où cette forme de réflexivité supérieure est accessible essentiellement à travers tout un processus d'échanges interindividuels, il importe au sociologue de susciter la conversation, de la soutenir et d'amener, par la parole, les individus à s'interroger sur eux-mêmes ${ }^{9}$. Cette pratique de la parole conduit les individus à entrer dans une forme $\mathrm{d}$ ' «autoanalyse provoquée et accompagnée » telle que décrite par Bourdieu (1993, p.1408). Questionnées sur leur vie, les personnes vont profiter de l'occasion que leur offre l'enquêteur, pour raconter leur quotidien et s'interroger sur elles-mêmes. En se livrant à des «commentaires continuels sur leurs activités » (Molénat, 2006, p.53), elles réalisent par là même un travail d'explicitation utile, voire salvateur en trouvant les mots pour exposer leurs souffrances et leurs malaises. Dans une certaine mesure, le sociologue contribue à créer les conditions favorables à cette auto-

\footnotetext{
${ }^{8}$ Pierre Bourdieu, pour qui les individus ne détiennent nullement la science infuse (1993, p.1413), explique lui aussi que ces derniers mobilisent au quotidien des connaissances pratiques ou ce qu'il appelle des « routines de la pensée ordinaire » (1994, p.9) leur permettant d'accéder à des réalités substantielles, c'est-à-dire tangibles et comme allant de soi (Ibid.). Notons que contrairement à Giddens, Bourdieu s'arrête là dans le degré de réflexivité, ne lui conférant, au-delà, qu'un caractère d'exceptionnalité, à l'occasion notamment de situations de crise.

${ }^{9}$ Bien entendu, Giddens est conscient que l'homme ne peut tout expliquer de façon verbale ni porter un regard lucide et cognitif sur toutes ses actions dont beaucoup ont des effets non intentionnels. Certaines formes de cognitions sont refoulées, enfouies dans un état inconscient ou limitées par la rationalité individuelle.
} 
analyse réflexive.

\subsection{LES CONTOURS DE LA SOCIOLOGIE D'INTERVENTION}

Débuter une enquête de terrain nécessite de respecter un ensemble de principes et une posture qui sont autant de conditions préalables pour débuter une analyse. La sociologie d'intervention répond à plusieurs critères fondamentaux avant, pendant et après l'analyse.

Premièrement, il ne peut y avoir d'intervention sans demande. Pour pouvoir investiguer un lieu, le sociologue doit être sûr que les acteurs en présence, ou tout au moins un nombre suffisant d'entre eux, expriment une réelle volonté de coopérer. La demande peut émaner des dirigeants - on parlera alors de commande ${ }^{10}$ - mais aussi des salariés confrontés à des situations de malaises, de tensions voire de conflits. Un travail d'explicitation de cette demande est d'ailleurs bien souvent nécessaire en amont de toute intervention. Deuxièmement, quand il arrive sur le terrain, le sociologue d'intervention doit nécessairement être pragmatique en considérant le sens commun comme producteur de savoirs utiles. Le principe qui le guide alors relève d'une certaine «philosophie de vie » donnant à l'acteur une place centrale dans la recherche de la vérité. Cette posture a amené des sociologues tels Luc Boltanski à préconiser le passage d'une sociologie critique bourdieusienne à une sociologie de la critique en attribuant au sociologue un rôle plus modeste dans l'interprétation des faits sociaux. Cette posture de la critique conduit à « recentrer l'attention du sociologue sur les acteurs en situation, en tant que principales agences de performation du social, au détriment d'une description cartographique du monde déjà là » (Boltanski, 2009, p.48). La maïeutique, qui fait référence à la mythologie grecque ${ }^{11}$, préconise d'amener les hommes à accoucher de leurs savoirs. Face à des acteurs en situation, notre conviction est que « personne n'est jamais qu'ignorance. Le savoir que chacun possède, qu'il affleure ou soit enfoui, ne demande qu'à

\footnotetext{
${ }^{10}$ Les commanditaires sont appelés par certains auteurs le «système-client ». Le premier à avoir introduit cette notion est R. Lippitt en 1947. Par cette expression, il entendait différencier l'origine réelle de la demande (pour cela, il identifiait quatre niveaux possibles : la personne individuelle, le groupe, l'organisation et la collectivité) et le système auquel les demandeurs appartenaient ou dont ils se réclamaient (voir Dubost, 1987, p. 190). Le mot client, quant à lui, exprime l'idée d'un service vendu et contractualisé avec un prestataire.

${ }^{11}$ La maïeutique fait référence à un personnage de la mythologie grecque : Maïa. Fille aînée des Pléiades et mère d'Hermès, Maïa était considérée par les grecs comme la déesse de l'accouchement et des sages femmes. Par analogie, le mot maïeutique renvoie à l'idée que tout homme peut, avec de la volonté et quelques dispositions intellectuelles, accoucher de ses savoirs cachés.
} 
être découvert » (Herreros 2009, p. 145). Ce retour vers l'intérieur, précise Friedberg, révèle une posture éminemment clinique, nécessairement contingente et rompant temporairement toute distance entre le sociologue et les acteurs en situation (1997, p.303). Il constitue une « abolition de la coupure épistémologique » entre la théorie et la pratique (1997, p.306), entre les savoirs savants et les savoirs communs. L'approche maïeutique accorde ainsi une importance toute particulière au sens commun considéré comme producteur de connaissances utiles à l'amélioration d'une situation ${ }^{12}$. Cette réhabilitation du sens commun dans l'analyse sociologique explique, du reste, la proximité que la sociologie d'intervention entretient avec la sociologique clinique. Cette ouverture, pour autant, ne va pas de soi dans la culture sociologique. Empreint des concepts de l'objectivité, du désenchantement, du positivisme et de la rupture épistémologique, le sociologue classique a des difficultés à prendre en compte la dimension psychique et psychologique des individus. Pourtant, explique Vincent De Gaulejac, «aller au plus près du vécu des acteurs conduit à remettre en question les frontières entre psychologie et sociologie, extériorité et intériorité, objectivité et subjectivité, réalité et représentation » (V. De Gaulejac, F. Hanique et P. Roche, 2007, p.54). L'individu est agent, acteur et sujet tout à la fois et simultanément selon le contexte et ses dispositions. Troisièmement, il convient, en situation d'intervention, d'être convaincu qu'aucune théorie ne se suffit à elle-même, ni qu'aucune méthode n'est, dans l'absolu, meilleure qu'une autre. Selon le contexte, une théorie sera plus utile qu'une autre, une méthode plus adaptée qu'une autre $^{13}$. Gilles Herreros propose d'adopter une posture méthodologique «plastique » (2004), refusant tout enfermement dans une méthode en particulier. A l'évidence, «la » méthode du sociologue d'intervention n'existe pas. Contre toute forme de «méthodolâtrie » (Herreros, 2004, p.147), les sociologues d'intervention affichent leur préférence pour une posture transdisciplinaire privilégiant le «relativisme méthodologique» et la «pensée métisse »

\footnotetext{
${ }^{12}$ Nous pensons, avec Anthony Giddens, que la relation entre la sociologie et les individus relève d'une «double herméneutique » (1994, p.24). D’une part, la science fournit des connaissances et des informations sur la vie sociale et remet en cause les «concepts des agents profanes» (Ibid.). D’autre part, ces connaissances forgées dans le métalangage des sciences sociales sont réinjectées dans la société et réappropriées par les acteurs. «Il y $a$ un va-et-vient entre l'univers de la vie sociale et le savoir sociologique et, dans ce processus, le savoir sociologique se modèle et remodèle l'univers social » (Ibid.).

${ }^{13}$ Dans un ouvrage récent (Bernardeau Moreau, 2013), l'auteur montre comment il est amené à convoquer l'analyse stratégique, la théorie de la contingence, la théorie de la régulation conjointe ou encore celle de la justification pour rendre compte de la spécificité de chacune de ses études de cas. Voir aussi l'ouvrage de Kaufmann sur l'entretien compréhensif (1996) où il préconise le recours, en certaines circonstances, à la « grounded theory » en partant des faits pour construire ensuite les hypothèses puis les cadres théoriques.
} 
(Herreros, 2004, p. 90). «A la discipline, nous préférons l'indiscipline transdisciplinaire » écrit Morin (1997, p. 160). A La méthode, optons pour «l'indiscipline méthodique» dit François Laplantine (postface Gilles Herreros, 2008, p. 245). Quatrièmement, la restitution des interprétations doit être réelle. Le sociologue doit accepter l'exercice inconfortable de la confrontation avec l'ensemble des acteurs concernés. La règle qui s'impose est « la règle du tout dire » avancée par les socioanalystes (voir Hess et Authier, 1994). Par le déploiement d'un débat ouvert et direct entre les chercheurs et les acteurs, la vraie vie de l'organisation avec ses propres codes, ses propres règles de fonctionnement, ses rapports de forces et de pouvoirs est amenée à se révéler aux yeux de tous sur la place publique. Certes, cette confrontation n'est pas aisée et fragilise le chercheur qui doit accepter de se mesurer à «l'indocilité des acteurs » (Vrancken, 1995) et être contesté et remis en cause.

\section{ETHIQUE DU LIEN ET VOLONTE A MINIMA DE NE PAS NUIRE}

Dans sa distinction bien connue entre l'éthique de la conviction et l'éthique de la responsabilité, Max Weber sépare le devoir d'agir des conséquences de l'action. L'homme agissant par conviction se préoccupe davantage de bien agir conformément aux normes et aux dogmes (religieux, sociaux ou autres). L'homme qui agit par responsabilité se soucie davantage des conséquences de son action dont il se sent redevable. L'éthique, nous dit de son côté Eugène Enriquez, désigne les grands principes d'orientation de notre conduite. Elle interroge chacun d'entre nous sur ce qu'il convient de faire. Elle nous pousse à prendre conscience du bien-fondé de nos actions, bien-fondé qui s'enracine dans une volonté $a$ minima de ne pas nuire (Enriquez, 2001). Modestement, le sociologue se propose d'agir sur la vie des individus dans les situations du quotidien. Son intention louable passe par la (re)construction du lien et l'émergence de savoirs utiles susceptibles de faire progresser les hommes en société. Son éthique personnelle est ce qui le guide dans sa démarche. Par son «principe activant» (Morin, 2004, p.16), elle vise à activer les liens entre les individus. Ce que Morin appelle «éthique de reliance », d'autres la dénomment éthique du lien, de la discussion, de la solidarité. Chez Richard Rorty, l'éthique est surtout une affaire de sensibilité à l'égard des autres, de leurs besoins et de leurs difficultés. Comme le suggère l'auteur, le sociologue doit surtout s'atteler à «minimiser les petites choses particulières qui nous

séparent» (Ibid., p.125). Il cherche moins les différences que les «mille petits traits» susceptibles de réunir les individus dans une «commune humanité » (Ibid., p.126). Perçue comme une morale personnelle, l'éthique du lien, explique Gilles Herreros, est le «butoir 
essentiel » (2009, p.237), le phare qui guide la route à l'horizon. Refusant de lui donner une définition à vocation universelle, Gilles Herreros la situe dans la posture générale du sociologue qui a intégré l'ensemble des dispositions et concepts développés par la sociologie d'intervention. Visant à l' «advènement du sujet $»^{14}$, l'éthique du sociologue se retrouve toute entière dans ce que l'auteur appelle l'anthropologie d'intervention. Par cette dénomination, il faut entendre les sciences de l'homme «débarrassées de leurs découpages disciplinaires» (Herreros, 2008, p.166). Pour se faire, le sociologue se fait médiateur et tiers. Pour les uns, il est tiers institué (Enriquez), pour les autres, il est tiers instruit (Serres) ou encore tiersintervenant (Herreros) ${ }^{15}$. A l'écoute permanente de l'acteur, il se veut pragmatique, puisant dans cette posture les ressorts de son utilité sociale.

\section{CONCLUSION : POUR UNE SOCIOLOGIE UTILE}

Dans sa thèse de 1979 qu'il consacre à la genèse de la sociologie d'intervention, Antoine Savoye en définit utilement quelques contours. Ainsi, il nous explique que la sociologie d'intervention est une sociologie de terrain où ce qui compte, «c'est le rapport direct institué entre le sociologue et la population qu'il étudie » (Savoye, 1979, p.3). L'individu, face à ses problèmes quotidiens, attend des sociologues qu'ils lui donnent des orientations et fournissent des éléments de réponses compréhensibles et exploitables. S'inspirant de la distinction faite par Chris Argyris entre connaissances applicables et connaissances utilisables, Michel Crozier explique que les premières sont celles qui permettent de comprendre une situation et les comportements des individus. En cela, elles sont nécessaires à ces derniers pour appréhender les problèmes et difficultés qu'ils rencontrent au quotidien. Les connaissances utilisables sont celles qui sont réellement mises en pratique de façon quasi expérimentale. Elles aboutissent à l'élaboration d'outils et de plans d'actions immédiatement exploitables par les acteurs du terrain. La difficulté, mainte fois soulignée par ailleurs en sciences sociales, est de passer des

\footnotetext{
${ }^{14}$ Par cette expression mobilisant tout à la fois les notions sociologiques, mais aussi psychologiques, voire philosophiques et psychanalytiques, il s'agit, pour le sociologue, non pas de créer le sujet mais plutôt d'apporter tous les éléments à sa mise à jour, à son éclosion et son déploiement (Herreros, 2008, p.114). Gilbert et Baldelli (2011), pour leur part, parlent de «surrection de l'acteur ». Dans le champ de l'intervention sociale, il s'agit de considérer que toute personne est capable d'être actrice de sa propre insertion dans la société.

15 La figure d'Hermès le messager, proposée par Michel Serres et Gilles Herreros, symbolise cette communication entre les sciences et les savoirs, ce passage entre les champs disciplinaires et les domaines d'activités.
} 
unes aux autres, de faire le lien entre la théorie et la pratique. Le problème, explique Michel Crozier, vient de ce que «la majeure partie de la recherche en sciences sociales est applicable, mais pas utilisable »(Crozier, 2000b, p. 292). La sociologie, il est vrai, peine souvent à apporter une aide concrète à l'individu en situation. Quand elle est interpelée par la demande sociale pour éclairer un problème, les réponses qu'elle propose ne sont pas toujours comprises et mobilisables dans l'action. Pour autant, face à ces constats, il n’y a pas de fatalité. La sociologie d'intervention a une double ambition : Elle vise à la fois à (re)légitimer les sciences sociales auprès d'acteurs sociaux qui les jugent parfois sévèrement, tant ils peuvent les trouver peu accessibles, voire hermétiques. Elle tente également de redonner du sens et de l'utilité à des sociologues qui, parfois, peinent à maintenir les liens, à la fois cognitifs et émotifs, entre eux et les individus qu'ils étudient, entre les concepts théoriques qu'ils manipulent et les réalités multiples d'un monde social en perpétuel mouvement.

\section{BIBLIOGRAPHIE}

ALTER N. 1995. «La légitimité de l'action sociologique en entreprise » in LEGRAND M., GUILLAUME J.-F.et VRANCKEN D., La sociologie et ses métiers, Paris, L’Harmattan, Logiques sociales. p. 143-149.

BERNARDEAU MOREAU D., 2013, La sociologie d'intervention. Le sociologue au cour des organisations associatives, sportives et de loisirs, Paris, L'Harmattan, Logiques sociales.

BOLTANSKI L., 2009, De la critique. Précis de sociologie de l'émancipation, Paris, Gallimard, Essais.

BOURDIEU P. (dir.), 1993, La misère du monde, Paris, Editions du Seuil, Points.

BOURDIEU P., 1994, Raisons pratiques. Sur la théorie de l'action, Paris, Seuil, Points Essais.

BOURDIEU P., 1997, Les usages sociaux de la science. Pour un sociologie clinique du champ scientifique, Paris, INRA, Editions.

BOUSSARD V., MERCIER D. et TRIPIER P., 2004, L'aveuglement organisationnel ou comment lutter contre les malentendus, Paris, CNRS Editions.

CASTEL R., 2004, « La sociologie et la réponse à la demande sociale », In LAHIRE B. (dir.), A quoi sert la sociologie ?, Paris, La Découverte.

COULON A., 2002 (1987), L'ethnométhodologie, Paris, PUF, Que sais-je ?.

CROZIER M., 2000a, A quoi sert la sociologie des organisations? Théorie, culture et 
société, Tome 1, Paris, Arslan.

CROZIER M., 2000b, A quoi sert la sociologie des organisations? Vers un nouveau raisonnement pour l'action, Tome 2, Paris, Arslan.

DUBAR C., 2006, Le pluralisme en sociologie : fondements, limites, enjeux, Socio-logos, $\mathrm{n}^{\circ} 1$, Varia, p. 1-14.

DUBET F., 2001, «Plaidoyer pour l'intervention sociologique », In VRANCKEN D. et KUTY O., La sociologie et l'intervention. Enjeux et perspectives, De Boeck Université, Ouvertures sociologiques, p. 89-110.

DUBET F., 2003, «Problèmes d'une sociologie de l'enseignement supérieur », In Felouzis G. (dir), Les mutations actuelles de l'université, Paris, PUF, p. 363-397.

DUBOST J., 1987, L'intervention psycho-sociologique, Paris, PUF, Sociologies.

DUBOST J., 2006, Analyse sociale et sociologies d'intervention, Paris, L'Harmattan, Histoire et mémoire de la formation.

ENRIQUEZ E., 2001, «L'éthique de l'intervenant», in VRANCKEN D. et KUTY O., La sociologie et l'intervention. Enjeux et perspectives, De Boeck Université, Ouvertures sociologiques, p.299-310.

FAURE S., SOULIE C. et MILLET M., 2005, Enquête exploratoire sur le travail des enseignants chercheurs. Vers un bouleversement de la table des valeurs académiques, Rapport d'enquête.

FELDER D., 2007, Sociologues dans l'action. La pratique professionnelle de l'intervention, Paris, L’Harmattan, Questions sociologiques.

FELOUZIS G., 2003, Les mutations actuelles de l'université, Paris, PUF.

FRIEDBERG E., 1997, Le pouvoir et la règle. Dynamique de l'action organisée, Paris, Points, Essais.

FRIEDBERG E., 1997, «La sociologie d'intervention est d'abord une sociologie », Gérer et comprendre, Annales des mines, $n^{\circ} 49$, septembre, p.93-94.

FRIEDBERG E., 2001, «Faire son métier de sociologue, surtout dans l'intervention », In VRANCKEN D. et KUTY O., La sociologie et l'intervention. Enjeux et perspectives, De Boeck Université, Ouvertures sociologiques, p. 111-132.

GARFINKEL H., 1967. Studies in ethnomethodology, Englewood cliffs, NJ. Prentice Hall.

GAULEJAC (De) V., HANIQUE F. et ROCHE P. (dir.), 2007, La sociologie clinique. Enjeux théoriques et méthodologiques, Paris, Eres, sociologie clinique.

GIDDENS A., 1987, La constitution de la société, Paris, PUF, Sociologies.

GIDDENS A., 1994, Les conséquences de la modernité, Paris, L'Harmattan, Théorie sociale 
contemporaine.

GILBERT Y. et BALDELLI B. (dir.), 2011, Intervention sociale et implication. Chercheurs, praticiens et usagers. Déconstruction, co-constructions et réciprocités, Perpignan, PUP, Etudes.

HERREROS G. 2001. «Sociologie d'intervention : Pour une radicalisation de quelques principes », In VRANCKEN D. et KUTY O., La sociologie et l'intervention. Enjeux et perspectives. De Boeck Université, Ouvertures sociologiques, p. 273-298.

HERREROS, G. 2004, "Sociologie d'intervention: sociologie plastique. Métis et métissage », Gérer et comprendre, Annales de l'Ecole des mines de Paris, n 75, mars.

HERREROS G., 2008, Au-delà de la sociologie des organisations. Sciences sociales et intervention, Paris, Erès, Sociologie économique.

HERREROS G., 2009, Pour une sociologie d'intervention, Toulouse, Erès, poche.

HESS R., 1981, La sociologie d'intervention, Paris, PUF, Le sociologue.

HESS R. et AUTHIER M., 1994, L'analyse institutionnelle, Paris, PUF, l'éducateur.

KAUFMANN J.-C., 1996, L'entretien compréhensif, Paris, Nathan.

LAHIRE B. (Sous la dir.), 2004, A quoi sert la sociologie?, Paris, La Découverte/Poche, Sciences humaines et sociales.

LEGRAND M., 1995, «Entre le piège et le défi d'une diversification des pratiques sociologiques », In LEGRAND M., GUILLAUME J.-F. et VRANKEN D., 1995, La sociologie et ses métiers, Paris, L'Harmattan, Logiques sociales, p.113-120.

MINGUET G., 2001, «Taxinomie de modèles sociologiques d'intervention », In VRANCKEN D. et KUTY O., La sociologie et l'intervention. Enjeux et perspectives, De Boeck Université, Ouvertures sociologiques, p. 19-68.

MOLENAT X., 2006, «L'individu réflexif, nouveau modèle sociologique?», Sciences humaines, $\mathrm{n}^{\circ} 175$, octobre, p.50-53.

MORIN E. (en collaboration avec Sami NAIR), 1997, Une Politique de civilisation, Paris, Arléa.

MORIN E., 2004, Ethique, Paris, Edition du Seuil.

PIRIOU O., 1999, Pour une sociologie des sociologues. Formation, identité, profession, Lyon, ENS Editions, Sociétés, espaces, temps.

PIRIOU O., 2006, La face cachée de la sociologie. A la découverte des sociologues praticiens, Paris, Belin, Perspectives sociologiques. 
RICOEUR P., 1997, «A la gloire de la phronesis », in CHATEAU J.-Y., La vérité pratique : Aristote, Ethique à Nicomaque, livre 6, Paris, Vrin, p.13-22.

RORTY R., 1995, L'espoir au lieu du savoir. Introduction au pragmatisme, Paris, Albin Michel.

SAINSAULIEU R., 1995, «Le métier de sociologue en pratique », In Legrand M., Guillaume J.-F.et Vrancken D., La sociologie et ses métiers, Paris, L'Harmattan, Logiques sociales. p. 13-34.

SAVOYE A., 1979, Genèse de la sociologie d'intervention, Thèse de doctorat de troisième cycle de sociologie, Université Paris X (fond documentaire du CEDIAS).

UHALDE M. (dir.), 2001, L’intervention sociologique en entreprise. De la crise à la régulation sociale, Paris, Desclée de Brouwer, Sociologie économique.

VELTZ P., 2007, Faut-il sauver les grandes écoles? De la culture de la sélection à la culture de l'innovation, Paris, Presse de Sciences Po.

VRANCKEN D., 1995, «Les tensions du métier de sociologue entre fondamentalisme et approche clinicienne », In LEGRAND M., GUILLAUME J.-F. et VRANCKEN D., La sociologie et ses métiers, Paris, L’Harmattan, Logiques sociales, p. 351-358.

VRANCKEN D., 2001, «L'intervention au cœur de la sociologie », In VRANCKEN G. et KUTY O. (Dir.), La sociologie et l'intervention. Enjeux et perspectives, De Boeck Université, Ouvertures sociologiques, p. 311-322.

VRANCKEN D. et KUTY O., 2001, La sociologie et l'intervention. Enjeux et perspectives. Paris, De Boeck Université, Ouvertures sociologiques. 